\title{
Editorial
}

\section{Adult vaccination for chronic disease}

Vaccination is a process where a person is made immune or resistant to certain type of infectious disease by administering vaccine - immune substance which stimulate body's own immune system to protect him against certain disease.

Vaccination is an imported part of health care management. Child vaccination in Bangladesh is very successful. Now we need to focus on adult vaccination specially for senior citizen as most of them are suffering from chronic disease like Diabetes,hypertension, ischemic heart disease, chronic kidney disease, chronic liver disease, immunocompromised diseases or chronic obstractive airway disease. This disease itself makes financial burden as well as some infectious diseases aggravates it more. Infectious diseases make these chronic diseases worse push these persons to be hospitalized which put his / her family a big economical instability.

In 429 BC Greek historian Thucydides first notice that people who survive small pox do not get reinfected and in 1796, Edward Jenner discovered small pox vaccine. In 1980 small pox is eradicated by WHO from world which is very successful program to eradicate a serious diseae from our community.

Now a days a lot of vaccine discovered to help to prevent infectious disease. Influenza vaccine which is yearly given to help chronic disease patient to prevent not to get worse yearly. Other vaccine like pneumococcal vaccine, varicella vaccine, hepatitis A, B vaccine, meningococcal vaccine, zoster vaccine need to be plan to give chronic diseases patient in a planned manner like EPI vaccination.

We need to make aware to chronic disease patient about vaccination as well as need as practice schedule for all clinical practitioner to reduce disease related economical burden to person, society and our country.

\section{Dr. Amal Kerishna Paul \\ Assistant Professor Department of Medicine Member, Editorial Board}

